\title{
ARTICLE Wetting of Laser Textured Cu Surface by Ethylene Glycol and Sn
}

\section{Kaibin Xie Yujie Ge Qiaoli Lin*}

State Key Laboratory of Advanced Processing and Recycling of Non-ferrous Metal, Lanzhou University of Technology, Lanzhou, 730050, China

\section{ARTICLE INFO}

Article history

Received: 3 July 2021

Accepted: 15 July 2021

Published Online: 20 July 2021

\section{Keywords:}

Wettability

Nanosecond laser

Roughness

Soldering

Texturing

\section{Introduction}

The roughness of the surface could be a factor which influences the wettability. For the purpose of objective characterization with good reproducibility of results, the smooth surface with very limited roughness is required, usually, in a range of $\sim 2-10 \mathrm{~mm}$, the roughness $\left(R_{a}\right)$ should be dozens of nanometers for the polished metallic surface, and dozens to hundreds of nanometers for the polished polycrystalline ceramic surface. On the other hand, with development of soldering and brazing technology, a sound joint is not just depended on the reaction at interface, and some uncertain failure may also be caused due to the brittleness of reaction products. The factitious microcosmic topography of surface may be a method to control wettability, and then the dependence of interfacial reaction could be alleviated.

Although the effect of roughness or surface topography

\begin{abstract}
The effect of microcosmic morphologies of textured $\mathrm{Cu}$ surface by nanosecond laser on the inert wetting and reactive wetting, i.e., ethylene glycol/copper and tin/copper wetting systems, was studied by using modified sessile drop methods. To create different surface roughness, the microcosmic morphologies with different spacing of grooves were constructed by nanosecond laser. The results showed that the inert wetting (ethylene glycol/copper) was consistent with Wenzel model, while the reactive wetting results deviated from the model. $\mathrm{In} \mathrm{Sn} / \mathrm{Cu}$ reactive wetting system, the interfacial evolution in the early stage and the pinning of triple line by the precipitated $\eta-\mathrm{Cu}_{6} \mathrm{Sn}_{5}$ caused the rougher surface and the worse final wettability. When the scale of artificial roughness exceeded the roughness that was caused by interfacial reaction after reaching the quasiequilibrium state at interface, the final wettability could be improved.
\end{abstract}

on the wettability has been developed by Wenzel model since $1936^{[1]}$, some controversies are still remained in a reactive wetting system, especially for the reactive wetting at high temperatures. As described by Wenzel model, the apparent contact angle can be predicted as

$\cos \theta_{\mathrm{a}}=w_{r} \cos \theta_{Y}$

where $\theta_{\mathrm{a}}$ and $\theta_{Y}$ are the apparent contact angle on the rough surface and the contact angle on the smooth ideal surface, and $w_{r}$ is the area ratio of rough surface to smooth surface. Theoretically, when $\theta_{Y}$ is below $90^{\circ}$, the increased roughness would improve wettability, and vice versa. However, in the reality, the apparent contact angles are more or less deviated from the predicted value of Wenzel model. Palasantzas et al. ${ }^{[2]}$ indicated that the critical transition angle was not $90^{\circ}$, and should be smaller than $90^{\circ}$, and further decreases with increasing a roughness exponent $\mathrm{H}$. Wu et al. ${ }^{[3]}$ suggested that the wettability of

*Corresponding Author:

Qiaoli Lin,

State Key Laboratory of Advanced Processing and Recycling of Non-ferrous Metal, Lanzhou University of Technology, Lanzhou, 730050, China;

Email:lqllinqiaoli@163.com 
the compound forming reactive wetting system would be worsening by a rough surface of substrate. Chen et al. ${ }^{[4]}$ developed a theoretical model and verified the wetting behavior experimentally. However, the conflict with Wu's viewpoint ${ }^{[3]}$ was raised, as predicted by Chen at al. ${ }^{[4]}$, the wettability in a reactive system could be promoted with less active addition. Also, Satyanarayan and Prabhu ${ }^{[5]}$ found that the rough surface can improve wettability in the wetting of SAC solders/Cu system, which is a typical reactive wetting system. In the study of $\mathrm{Al} / \mathrm{h}$-BN reactive wetting system, Shen et al. ${ }^{[6]}$ considered the different roughness of h-BN surface was the key factor which caused the reported contact angles were so scattered, and also showed the smoother surface and the smaller contact angle, i.e., the wettability would be worsen by the increased surface roughness. Further, Chen and Duh ${ }^{[7]}$ also showed the wettability of solders would be degraded as the substrates become rough in the wetting of $\mathrm{Sn}-\mathrm{Bi} / \mathrm{Cu}$ system. After investigating about the wetting of sixteen materials combinations at room temperature and high temperatures with the selection of atomic liquid (metallic liquid) and molecular liquid (organic liquid), Hitchcock et al. ${ }^{[8]}$ suggested wettability would be deteriorated by roughening a substrate whether of wetting $\left(\theta_{Y}<90^{\circ}\right)$ and non-wetting $\left(\theta_{Y}>90^{\circ}\right)$ system. In the study of the reactive wetting of $\mathrm{Cu}-\mathrm{Sn}-\mathrm{Ti}$ and $\mathrm{Ag}-\mathrm{Cu}-\mathrm{Ti}$ alloys on $\mathrm{SiC}$, Tillmann et al. ${ }^{[9]}$ showed no distinct correlation between roughness and wettability. Kramer et al. ${ }^{[10]}$ tried to use Cassie-Baxter model explained the effect of microtextured surface topography on the wetting of In and Sn film by liquid Ga-In alloys, but found that the results only showed a good agreement prior to reactive wetting. In the reactive wetting of $\mathrm{CuAgTi} / \mathrm{alumina}$ system, the results were obtained by Voytovych et al. ${ }^{[11]}$, indicated that the wetting did not vary significantly with the roughness of the solid. To sum up, whether roughness of the surface would promote or deteriorate wettability in a reactive wetting system at high temperature is still ambiguous, as well as the factor caused divergence.

In this work, the polished $\mathrm{Cu}$ surface was textured by nanosecond laser. The liquids, ethylene glycol and pure $\mathrm{Sn}$, were selected, which has the similar contact angles on $\mathrm{Cu}$ surface, and also the comparison between molecular and atomic liquids were considered. The textured $\mathrm{Cu}$ surface with different roughness was studied. The purpose of this work is to reveal the key factors on the wettability.

\section{Experimental Procedure}

The substrates of $\mathrm{Cu}$ were in a purity of $>99.99 \%$ and dimension of $20 \mathrm{~mm} \times 20 \mathrm{~mm} \times 5 \mathrm{~mm}$. Ethylene glycol and Sn with a purity of $>99.9 \%$ and $>99.999 \%$ were selected.
The surface of $\mathrm{Cu}$ substrate was firstly polished to the roughness $\left(\mathrm{R}_{\mathrm{a}}\right.$, the mean deviation in contour arithmetic) of $\sim 30-50 \mathrm{~nm}$ in a tested distance of $2 \mathrm{~mm}$, and then, some substrates were selected for surface laser texturing. The substrates were degreased with acetone and dried by blowing dry air, and then textured by HY-TS20A fiber nanosecond laser machining. The textured morphology was further characterized by infinite focus optical 3D surface metrology (Infinite Focus G4, Alicona Imaging $\mathrm{GmbH}$, Austria).

For the wetting of ethylene glycol/Cu, the experiments were performed by optical surface analyzer (OSA60, NBSI, China); For the wetting of $\mathrm{Sn} / \mathrm{Cu}$, because of oxygen partial pressure of atmosphere, the wetting experiments were performed under a high vacuum $(\sim$ $3-4 \times 10^{-4} \mathrm{~Pa}$ ) by designed device, as described in detail elsewhere ${ }^{[12]}$. The improved sessile drop method was adopted for the study of $\mathrm{Sn} / \mathrm{Cu}$. The advantage of this method, especially for a metallic system, the effect of original oxide film can be alleviated, i.e., the substrate was annealed at high temperature under a high vacuum before wetting. In the vacuum chamber, the substrate was placed on the holder horizontally, and metallic block was stored in a stainless-steel tube outside heating zone. The chamber was vacuumed to a high vacuum $\left(\sim 5-7 \times 10^{-4}\right.$ $\mathrm{Pa}$ ), and then the substrate was heated to $700{ }^{\circ} \mathrm{C}$ at a rate of $20{ }^{\circ} \mathrm{C} / \mathrm{min}$, and dwelling for $10 \mathrm{~min}$, finally cooled to the experimental temperatures. After such a treatment, the covered oxide film on substrate can be broken, and the wetting behavior would be close to the intrinsic behavior. As the experimental temperature was stable, the drop was transferred to the surface of substrate. Meanwhile the camera recorded the variation of the whole wetting process.

After wetting experiments, the profiles of drop were analyzed by Surface Meter Elements analysis software (developed by BNSI company, China) to calculate the contact angle and drop base radius. Selected wetting couples were sectioned and polished for microstructural observation using a scanning electron microscope (SEM, FEG 450, Netherlands) equipped with energy dispersive spectrometers (EDSs) in a spot size of $\sim 2 \mathrm{~mm}$ and an optical microscope (OM, MEF-3, Austria) for analysis.

\section{Results and Discussion}

The square texture with different spaces was constructed by nanosecond laser. The spaces are 50 $\mathrm{mm}, 75 \mathrm{~mm}, 85 \mathrm{~mm}$ and $100 \mathrm{~mm}$. The typical textured surface of space $\sim 85 \mathrm{~mm}$ as well as the three-dimensional morphology were shown in Figures 1 (a) and (b). Because of the texturing process by spot laser, the structure was 
similar with tight stamp hole. The depth, after laser ablation, is in $\sim 1-2 \mathrm{~mm}$, which is much smaller than Chen's work ${ }^{[4]}$, but the spaces between two stripes are wider. By varying the space, the different roughness can be obtained, as shown in Figure 1(c). The linear variation of space with roughness indicates the regular controlled morphologies can be obtained by nanosecond lasers.

In the inert wetting system, i.e., the wetting of ethylene glycol/Cu system, the contact angles of ethylene glycol on polished $\mathrm{Cu}$ surface are almost invariant, are $\sim 34.1^{\circ}$, as shown in Figure 2(a). With increase of roughness (or decrease of line spacing by ablation), the pinning of triple line moving is obvious. The decrease of contact angles becomes slow also monotonous, which always take several seconds until reached final contact angle. Based on the obtained actual morphology, the area rate in Equation (1), $w_{r}$, can be calculated. The final contact angle vs. $w_{r}$ has a good linear relationship, as shown in Figure 2(b). The intercept, when $w_{r}=1$, the intrinsic contact angle for ethylene glycol on ideal smooth $\mathrm{Cu}$ surface can be deduced is $\sim 36^{\circ}$. Also, the limitation of $w_{r}$ can be calculated for perfect wetting, is $\sim 1.33$. When $w_{r}$ is larger than that value, the wetting type may change from Wenzel model to Cassie model (heterogeneous wetting with air).
The results of the wetting of ethylene glycol/Cu show a good agreement with Wenzel model, i.e., the wettability can be improved definitely with the increase of roughness.

For the reactive wetting, $\mathrm{Sn} / \mathrm{Cu}$ system, so many complex factors would cause the scattered data. The oxide film of the substrate and interfacial reaction are the main factors. As shown in Figure 3(a), the wetting behavior was influenced by the initial conditions, significantly. Without pre-heat treatment before dropping, the droplet was transferred when temperature was stable at $250{ }^{\circ} \mathrm{C}$, the non-wetting of initial contact angle $\left(\sim 112^{\circ}\right)$ can be received as well as the slow spreading velocity. As the substrate was first heated to $450{ }^{\circ} \mathrm{C}$ at a rate of $20^{\circ} \mathrm{C} / \mathrm{min}$ and isothermal dwelling for $10 \mathrm{~min}$, and then cooled to $250{ }^{\circ} \mathrm{C}$, the initial contact angle was decreased to $\sim 68^{\circ}$, and also the spreading was accelerated. After anneal at $700{ }^{\circ} \mathrm{C}$ for $10 \mathrm{~min}$, the effect of oxide film on the substrate can be relieved. The final contact angles almost do not depend on the anneal process, are $\sim 34^{\circ}$, which may due to the high reactivity between $\mathrm{Cu}$ and $\mathrm{Sn}$. The effect of surface texturing morphology, as shown in Figure 3(b), the textured morphology would cause a poor wettability, i.e., the larger initial and final contact angles comparing to polished one, also the spreading was slowing down.
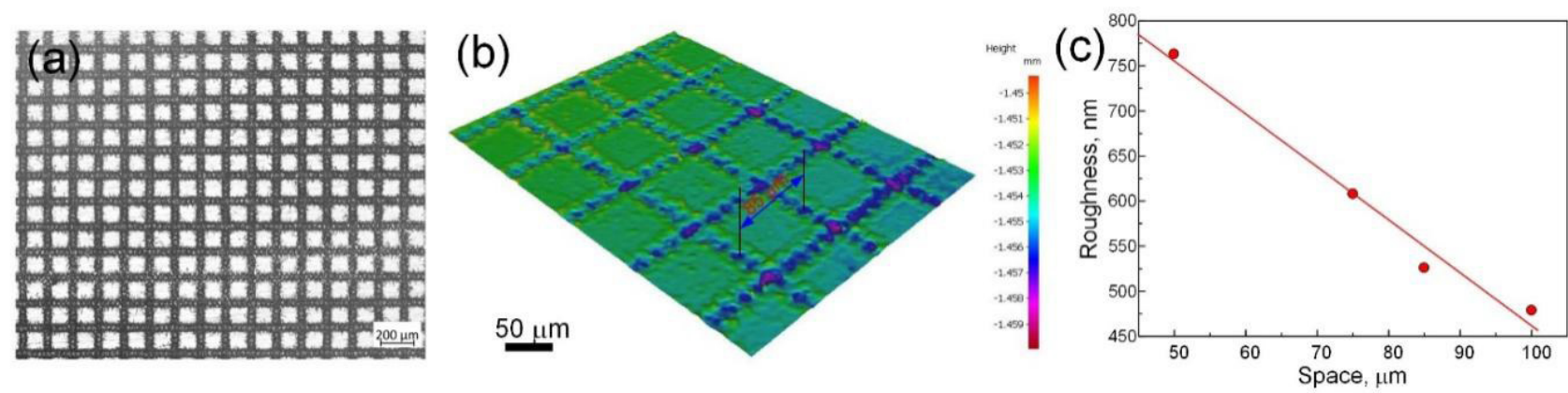

Figure 1. Images of $\mathrm{Cu}$ substrate surface by nanosecond laser textured (a) 2D profile and (b) 3D profile; (c) Roughness as a function of space for nanosecond laser textured.
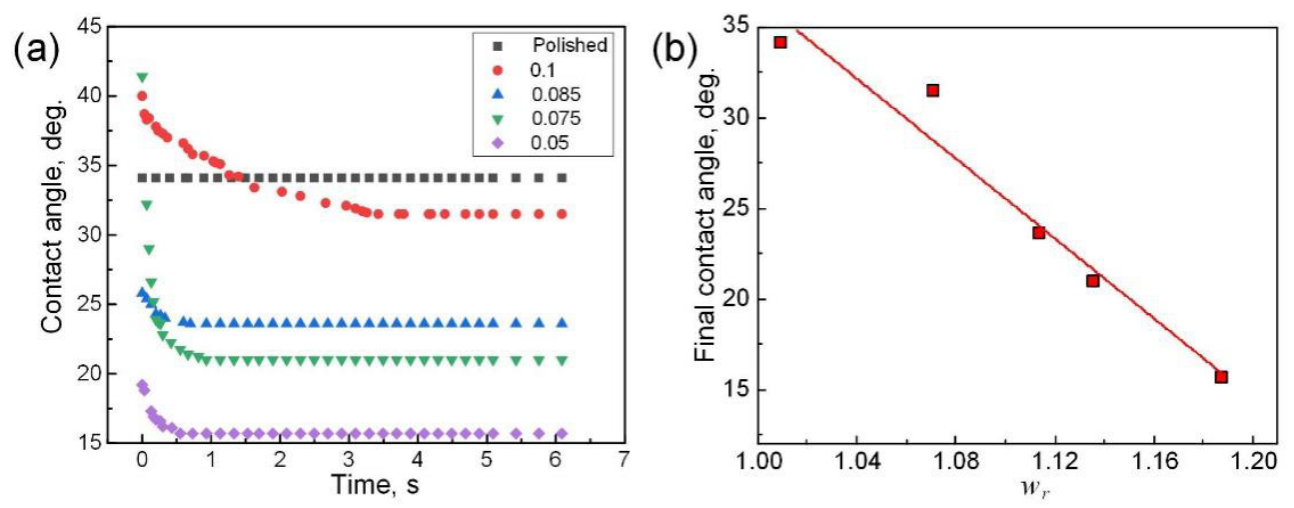

Figure 2. (a) Variations with time of the contact angle of ethylene glycol spreading on textured $\mathrm{Cu}$ substrate with different space at room temperature; (b) Final contact angle as a function of $w_{r}$ for ethylene glycol/textured Cu system. 
Obviously, the abnormal phenomenon is inconsistent with Wenzel model, as shown in Figure 3(c). The rougher the surface caused the poor wettability in the $\mathrm{Sn} / \mathrm{Cu}$ reactive wetting system.

The cross-sectional microstructures of the interface were shown in Figure 4. The typical continuous bilayer structures can be observed. Based on the EDS results, $\mathrm{Cu}_{3} \mathrm{Sn}$ layer is the layer contacted with $\mathrm{Cu}$, and $\mathrm{Cu}_{6} \mathrm{Sn}_{5}$ layer is the layer contacted with liquid. The textured morphology cannot be distinguished in all structures. As be mentioned above, the textured micro-groove in a depth only $\sim 1-2 \mathrm{~m}$, which can be easily covered up during reaction.

The deviation from Wenzel model should also be related to the interfacial reaction. Chen et al ${ }^{[4]}$. proposed a model, for the "non-composite surface" case (Sn-based solder/Cu case), the variation of total energy can be expressed as the increased area of interface multiply the interfacial tension. However, the model is suitable only for inert system. As a reactive wetting system, the interfacial area is varying during reaction as well as interfacial tension. The first stage of the reaction at interface is the dissolution, i.e., the dissolution of $\mathrm{Cu}$ into liquid. The laser textured micro-grooves would become flat by dissolution due to the sharp-angled effect, meanwhile, the precipitation of reaction product (IMCs) would further cause the interface become flat. The critical nucleation radius $r^{*}$ of the precipitation of $\eta-\mathrm{Cu}_{6} \mathrm{Sn}_{5}$ or $\varepsilon-\mathrm{Cu}_{3} \mathrm{Sn}$ can be expressed as,

$r^{*}=2 \sigma_{\eta(\varepsilon) \mathrm{L}} T_{\mathrm{e}} / L_{\mathrm{e}} \Delta T$

where $T_{\mathrm{e}}$ is the equilibrium precipitation temperature, $680 \mathrm{~K}$ for $\eta$ phase and $871 \mathrm{~K}$ for $\varepsilon$ phase ${ }^{[13]}, L_{\mathrm{e}}$ is the fusion heat per unit volume $\left(8.69 \times 10^{8} \mathrm{~J} / \mathrm{m}^{3}\right.$ for $\eta$ phase and $8.01 \times 10^{8} \mathrm{~J} / \mathrm{m}^{3}$ for $\varepsilon$ phase $\left.{ }^{[13]}\right), \Delta T$ is the undercooling of $\eta$ or $\varepsilon$ phase and $\sigma_{\eta(\varepsilon) \mathrm{L}}$ is the interface energy between precipitated phase and liquid solder $\left(0.09214 \mathrm{~J} / \mathrm{m}^{2}\right.$ for $\sigma_{\eta \mathrm{L}}$ and $0.1659 \mathrm{~J} / \mathrm{m}^{2}$ for $\left.\sigma_{\varepsilon \mathrm{L}}{ }^{[14]}\right) . r^{*}$ can be calculated, is $\sim 0.9-1$ $\mathrm{nm}$ which is much smaller than the scale of laser textured roughness. On the other hand, for a heterogeneous nucleation, the micro-groove (concave interface) would decrease the Gibbs free energy for nucleation, and then increase the nucleation rate. Therefore, the micro-groove would be filled IMCs due to the preferred nucleation, and then made the interface become flat. This stage took place only in milliseconds ${ }^{[15]}$. Therefore, in the early stage, the interface with the smaller roughness is much easier to be smooth. The smoother surface and the lower thermodynamic barrier for nucleation for the initial stage, which induced better initial wettability.

However, the roughness caused by the precipitated phase may further cover up the original laser textured morphology. When the size or thickness of grown precipitated phase was over the scale of original roughness, the wettability might be determined by the roughness of precipitated phase $\left(\eta-\mathrm{Cu}_{6} \mathrm{Sn}_{5}\right.$
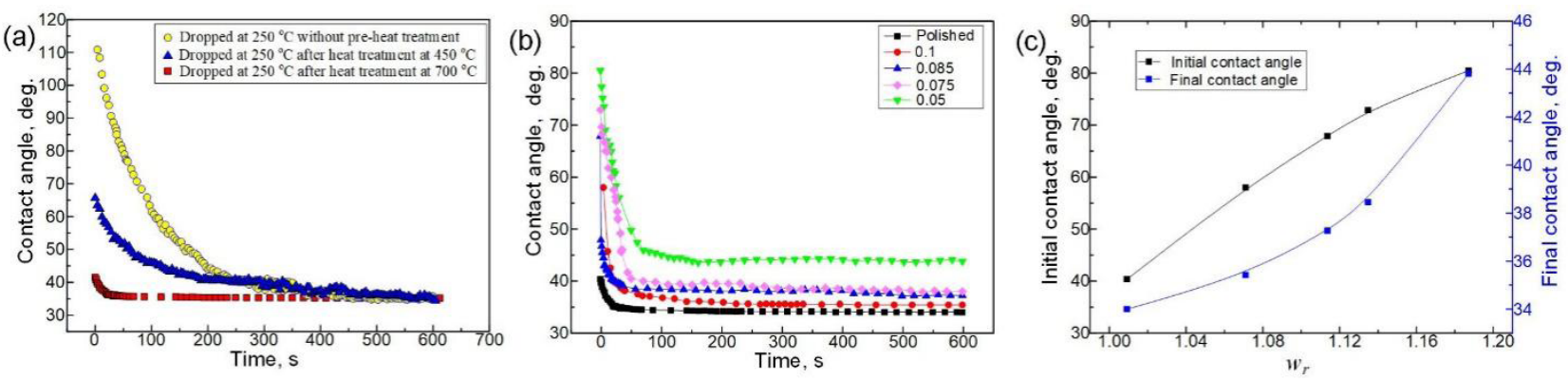

Figure 3. Variations with time of the contact angle of $\mathrm{Sn}$ droplet spreading on $\mathrm{Cu}$ substrate (a)with different pre-heat treatment temperature and (b)with different space at $250{ }^{\circ} \mathrm{C}$; (c) Initial contact angle and final contact angle as a function of $w_{r}$ for $\mathrm{Sn} /$ textured $\mathrm{Cu}$ system.
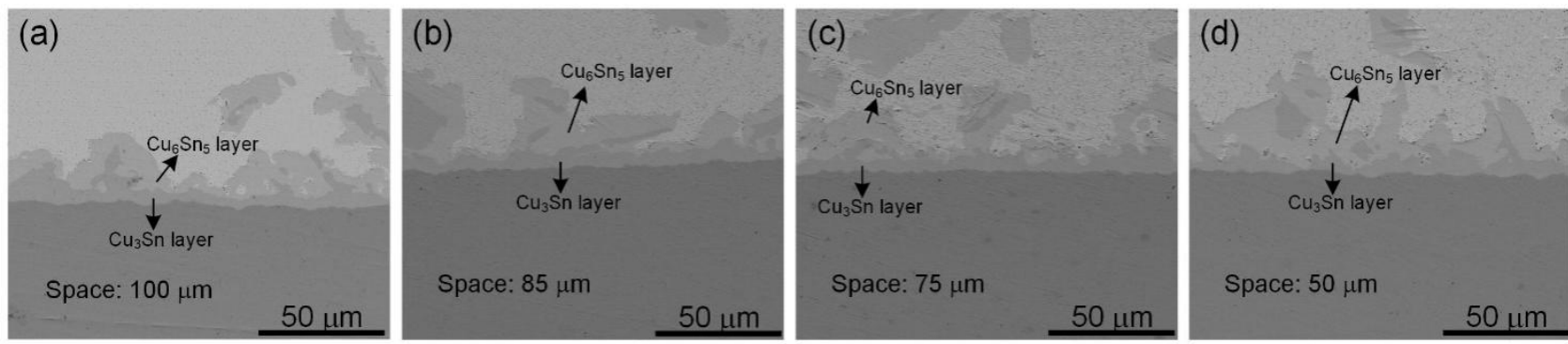

Figure 4. Cross section micro topography of wetting Sn drop on $\mathrm{Cu}$ substrate with difference space: (a) $100 \mathrm{~mm}$; (b) 85 $\mathrm{mm}$; (c) $75 \mathrm{~mm}$; (d) $50 \mathrm{~mm}$. 
phase). The growth kinetics of Sn-Cu IMCs can be described by, $D \approx t^{\mathrm{n}}$, where $D$ is the thickness of $\eta-\mathrm{Cu}_{6} \mathrm{Sn}_{5}$ layer, and $n \sim 1 / 3$ which is related to grain boundary diffusion and limited by the coarsening of the microstructure and elimination of fast mass diffusion paths ${ }^{[16]}$. In 100s (the wetting is almost in equilibrium), the thickness of $\eta-\mathrm{Cu}_{6} \mathrm{Sn}_{5}$ layer is $\sim 4.6 \mathrm{~m}$, which is larger than the depth after laser ablation. And then, the roughness was determined by the precipitated $\eta-\mathrm{Cu}_{6} \mathrm{Sn}_{5}$ phase. Therefore, the final wettability was weak dependent on the original roughness in this study. When the scale of artificial roughness exceeded the roughness that was caused by interfacial reaction after reaching the quasi-equilibrium state at interface, the final wettability may be improved by texturing process before wetting.

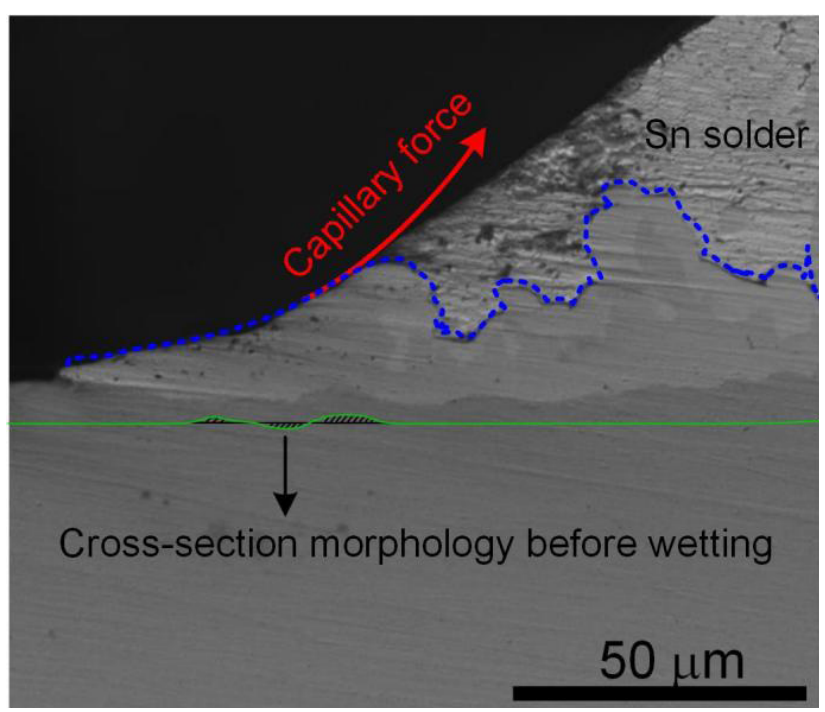

Figure 5. Cross section of the triple line region of $\mathrm{Sn} / \mathrm{Cu}$ wetting system

On the other hand, the rougher surface would cause the higher probability for the pinning of triple line. The typical configuration at the position close to triple line was shown in Figure 5. The triple line was stopped at the edge of $\eta-\mathrm{Cu}_{6} \mathrm{Sn}_{5}$ layer. The shaded area of the green solid line in Figure 5 is the cross-sectional morphology after laser textured. As can be seen, although the original laser textured morphology would be covered up by the nucleation of IMC in the early stage (as mentioned above), the significant mass transfer reaction at that position under the capillary force would prompt the growth of IMCs to the perpendicular direction, and then form a barrier that prevents the triple line from moving. Finally, the triple line was pinned by $\eta-\mathrm{Cu}_{6} \mathrm{Sn}_{5}$ layer. Therefore, the rougher surface and the worse final wettability can be correlated.

\section{Conclusions}

In this study, Wenzel model was verified by the wetting of laser textured $\mathrm{Cu}$ surface by ethylene glycol and tin, as the inert and reactive wetting systems. The obtained results indicated that the inert wetting system had a good agreement with Wenzel model. However, in a reactive wetting system, the rougher surface caused the worse wettability, which is inconsistent with Wenzel model. In the early stage of $\mathrm{Sn} / \mathrm{Cu}$ system, the fast nucleation process and the small nucleation radius at interface would make the interface smooth. Therefore, the smoother surface and the better initial wettability can be correlated. When the scale of artificial roughness exceeded the roughness that was caused by interfacial reaction after reaching the quasi-equilibrium state at interface, the final wettability may be improved by texturing process before wetting. The enhanced mass transfer reaction in the laser ablation zone under the capillary force at the triple line would prompt the growth of IMCs to the perpendicular direction, and then pin of triple line. Therefore, the rougher surface and the worse final wettability can be correlated.

\section{Credit Authorship Contribution Statement}

Kaibing Xie: Methodology, writing, original draft. Yujie Ge: Data analysis, Editing. Qiaoli Lin: Supervision, Work idea, Revision.

\section{Declaration of Competing Interest}

The authors declare that they have no known competing financial interests or personal relationships that could have appeared to influence the work reported in this paper.

\section{Acknowledgement}

This work is supported by National Natural Science Foundation of China (Nos. 51665031).

\section{Conflict of Interest}

No conflict of interest exists to declare.

\section{References}

[1] R.N. Wenzel, Resistance of solid surfaces to wetting by water, Transactions of the Faraday Society. 28(1936) 988-994.

[2] G. Palasantzas, J. T. M. D. Hosson, Wetting on rough surfaces, Acta Materialia. 49(2001) 3533-3538.

[3] M. Wu, L.L. Chang, L. Zhang, X.B. He, X.H. Qu, Effects of roughness on the wettability of high temperature wetting system, Surface and Coatings Technology. 287(2016) 146-152.

[4] H.Y. Chen, J.K. Peng, L. Fu, X.C. Wang, Solder wet- 
ting behavior enhancement via laser-textured surface microcosmic topography, Applied Surface Science. 368(2016) 208-215.

[5] Satyanarayan, K.N. Prabhu, Spreading behavior and evolution of IMCs during reactive wetting of SAC solders on smooth and rough copper substrates, Journal of Electronic Materials. 42(2013) 2696-2707.

[6] P. Shen, H. Fujii, K. Nogi, Effect of temperature and surface roughness on the wettability of boron nitride by molten Al, Journal of Materials Science. 42(2007) 3564-3568.

[7] Y.Y. Chen, J.G. Duh, B.S. Chiou, The effect of substrate surface roughness on the wettability of Sn-Bi solders, Journal of Materials Science: Materials in Electronics. 11(2000) 279-283.

[8] S.J. Hitchcock, N.T. Carroll, M.G. Nicholas, Some effects of substrate roughness on wettability, Journal of Materials Science. 16(1981) 714-732.

[9] W. Tillmann, J. Pfeiffer, L. Wojarski, Influencing Factors on the Reactive Wetting of $\mathrm{Cu}-\mathrm{Sn}$-Ti-and Ag-CuTi-Alloys on Silicon Carbide-Microstructural Observations, Effects and Multivariate Modelling, Advances in Science and Technology. 88(2014) 172-177.

[10] R.K. Kramer, J.W. Boley, H.A. Stone, J.C. Weaver, R.J. Wood, Effect of microtextured surface topogra- phy on the wetting behavior of eutectic gallium-Indium alloys, Langmuir. 30 (2014) 533-539.

[11] R. Voytovych, F. Robaut, N. Eustathopoulos, The relation between wetting and interfacial chemistry in the $\mathrm{CuAgTi}$ /alumina system, Acta Materialia. 54(2006) 2205-2214.

[12] Q.L. Lin, P. Shen, L. Yang, S. Jin, Q. Jiang, Wetting of TiC by molten $\mathrm{Al}$ at $1123-1323 \mathrm{~K}$, Acta Materialia. 59(2011) 1898-1911.

[13] W. Zhai, W.L. Wang, D.L. Geng, B. Wei, A DSC analysis of thermodynamic properties and solidification characteristics for binary $\mathrm{Cu}-\mathrm{Sn}$ alloys, Acta Materialia. 60(2012) 6518-6527.

[14] W. Zhai, B. Wei, Direct nucleation and growth of peritectic phase induced by substantial undercooling condition, Materials Letters. 108(2013) 145-148.

[15] R.A. Gagliano, G. Ghosh, Fine, Nucleation kinetics of $\mathrm{Cu}_{6} \mathrm{Sn}_{5}$ by reaction of molten tin with a copper substrate, Journal of Electronic Materials. 31(2002) 1195-1202.

[16] N. Dariavach, P. Callahan, J. Liang, R. Fournelle, Intermetallic growth kinetics for $\mathrm{Sn}-\mathrm{Ag}, \mathrm{Sn}-\mathrm{Cu}$, and $\mathrm{Sn}-\mathrm{Ag}-\mathrm{Cu}$ lead-free solders on $\mathrm{Cu}, \mathrm{Ni}$, and $\mathrm{Fe}-42 \mathrm{Ni}$ substrates, Journal of Electronic Materials. 35(2006) 1581-1592. 